\title{
Single cell assessment of yeast metabolic engineering for enhanced lipid production using Raman and AFM-IR imaging
}

\author{
Kamila Kochan ${ }^{1 \dagger}$, Huadong Peng ${ }^{2 \dagger}\left(\mathbb{0}\right.$, Bayden R. Wood ${ }^{1}$ and Victoria S. Haritos ${ }^{2 *}$
}

\begin{abstract}
Background: Biodiesel is a valuable renewable fuel made from derivatized fatty acids produced in plants, animals, and oleaginous microbes. Of the latter, yeasts are of special interest due to their wide use in biotechnology, ability to synthesize fatty acids and store large amounts of triacylglycerols while utilizing non-food carbon sources. While yeast efficiently produce lipids, genetic modification and indeed, lipid pathway metabolic engineering, is usually required for cost-effective production. Traditionally, gas chromatography (GC) is used to measure fatty acid production and to track the success of a metabolic engineering strategy in a microbial culture; here we have employed vibrational spectroscopy approaches at population and single cell level of engineered yeast while simultaneously investigating metabolite levels in subcellular structures.
\end{abstract}

Results: Firstly, a strong correlation $\left(r^{2}>0.99\right)$ was established between Fourier transform infrared (FTIR) lipid in intact cells and GC analysis of fatty acid methyl esters in the differently engineered strains. Confocal Raman spectroscopy of individual cells carrying genetic modifications to enhance fatty acid synthesis and lipid accumulation revealed changes to the lipid body (LB), the storage organelle for lipids in yeast, with their number increasing markedly (up to tenfold higher); LB size was almost double in the strain that also expressed a LB stabilizing gene but considerable variation was also noted between cells. Raman spectroscopy revealed a clear trend toward reduced unsaturated fatty acid content in lipids of cells carrying more complex metabolic engineering. Atomic force microscopy-infrared spectroscopy (AFM-IR) analysis of individual cells indicated large differences in subcellular constituents between strains: cells of the most highly engineered strain had elevated lipid and much reduced carbohydrate in their cytoplasm compared with unmodified cells.

Conclusions: Vibrational spectroscopy analysis allowed the simultaneous measurement of strain variability in metabolite production and impact on cellular structures as a result of different gene introductions or knockouts, within a lipid metabolic engineering strategy and these inform the next steps in comprehensive lipid engineering. Additionally, single cell spectroscopic analysis measures heterogeneity in metabolite production across microbial cultures under genetic modification, an emerging issue for efficient biotechnological production.

Keywords: Lipid bodies, Spectroscopy, Metabolic engineering, Heterogeneity, Triacylglycerol, Fatty acids

\section{Background}

Biodiesel is a versatile renewable fuel composed of fatty acid methyl esters produced from animal and plant

\footnotetext{
*Correspondence: victoria.haritos@monash.edu

${ }^{\dagger}$ Kamila Kochan and Huadong Peng contributed equally to this work

${ }^{2}$ Department of Chemical Engineering, Monash University, Clayton

Campus, Clayton, VIC 3800, Australia

Full list of author information is available at the end of the article
}

lipids-triacylglycerols (TAGs) [1, 2]. Microbial oils also have advantages as a source of TAG: microorganisms can be grown on non-food sugars, are minimally affected by seasons, generally robust and have short life cycles [1]. Many types of oleaginous eukaryotic species accumulate TAG in lipid bodies (LB), intracellular organelles considered primarily as storage vesicles for neutral lipids, in amounts up to $70 \%$ of total DCW biomass $[1,3,4]$. 
However, for cost-effective and high yielding production of TAG in oleaginous organisms such as yeast, metabolic pathway engineering and maximizing yield across the cultures is necessary $[1,3,5,6]$.

The metabolic engineering strategies for improvement of lipid production include up and down regulation of genes involved in the main steps of yeast lipid production: fatty acid (FA) biosynthesis, lipid accumulation and sequestration $[1,7]$. Increased fatty acid production can be achieved by higher expression of aldehyde dehydrogenase (ALD), acetyl-CoA synthetase (ACS) [8] and acetyl-CoA carboxylase (ACC) $[9,10]$ and FA accumulation in TAG is catalyzed by diacylglycerol acyltransferase (DGAT) [7] and other acyltransferases. Heterologous expression of highly active acyltransferases such as the DGAT1 from Arabidopsis thaliana has been shown to increase lipid yield in the yeast Saccharomyces cerevisiae [11] and similarly, LB stabilization proteins such as caleo$\sin$ (AtClo1) [12] have been shown to support LB formation. Down regulation of lipid mobilization enzymes such as the major TAG lipase, Tgl3, in S. cerevisiae, can also increase lipid content of cells [13].

Another factor affecting the productivity of microbially produced lipids is heterogeneity in individual cell performance across a culture [14]. Some of the variance is due to the stochastic nature of biological processes within gene regulation, transcription and translation, but response can be further distorted by variable responses to environmental conditions [15] including genetic engineering. While heterogeneity in cellular processes among isogenic microbial cells in a population has been known for some time, recent developments in visualization technologies have allowed measurement and monitoring of metabolite production both of populations and single cells within.

The formation and growth of LBs in cells are most commonly analysed by fluorescence imaging or histochemical staining [16]; these enable the visualization of LBs in multiple cells at once, but without providing details of their chemical composition. Vibrational spectroscopy-based techniques such as infrared (IR) and Raman spectroscopy (RS) are used in combination with digital imaging to enable rare insights into single cells of microalgae and yeast [17-20]. In particular, confocal Raman spectroscopy (CRS) has revealed intracellular structures such as LBs, due to its high spatial resolution falling in the range of few hundred $\mathrm{nm}$ depending on the excitation wavelength [21] and chemical composition information of selected intracellular structures $[18,19,22]$. The spatial resolution of imaging with the use of conventional IR spectroscopy is restricted by wavelength diffraction spatial resolution limit $(\sim 5.5 \mu \mathrm{m})$, making it less suitable for the study of intracellular structures of microorganisms.
Recently, however, a novel technique has been developed, based on a combination of IR spectroscopy and Atomic Force Microscopy (AFM-IR). In AFM-IR, IR absorption spectrum is acquired indirectly, by measuring the resulting thermal expansion of the sample. This approach overcomes the limitation of conventional IR-based imaging and achieves a spatial resolution of $\sim 100 \mathrm{~nm}$, thus enabling analysis of cellular constituents at the single cell level.

The aims of this research were to determine the effectiveness of metabolic pathway engineering approaches for enhanced lipid content in yeast through an examination of subcellular structures including LBs and to examine the heterogeneity of response in S. cerevisiae yeast cultures via vibrational spectroscopic approaches. We measured the overall changes in lipid content and other metabolites at a population level in whole cells by ATRFTIR following the introduction of genetic modifications, then undertook detailed studies of subcellular structures and changes in their chemical composition in single cells using CRS and AFM-IR. These approaches provided detailed, single cell information on subcellular structures at high-resolution for the engineered strains and also allowed representative sampling of the population for these traits. The increase in the total lipid content of engineered cells demonstrated in bulk samples reflected either a greatly increased number or greatly increased size of LBs; the increased size was likely due to the influence of the LB stabilizing protein, caleosin, expressed in these cells. In engineered yeast, LB fatty acid composition shifted towards lower content of UFA relative to SFA in the highly lipidic strains and cytoplasmic carbohydrate stores were heavily reduced. Vibrational spectroscopy analysis of yeast cells revealed unprecedented information on the effectiveness and effects of metabolic engineering strategies for higher lipid content that will also guide future approaches to the field.

\section{Methods \\ Yeast cell lines and culture conditions}

Four engineered strains of $S$. cerevisiae were evaluated in the study and compared with a control strain $(\mathrm{CON})$

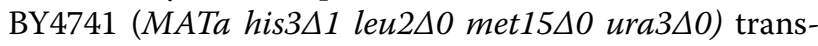
formed with three empty vectors. Genes selected for metabolic engineering are given in Table 1; the full description of the metabolic engineering strategy and selection and the combination of genes and expression plasmids are given in Ref. [23]. Expression of the introduced genes AtDGAT1, SEACS ${ }^{L 641 P}, A C C 1^{\text {S659A, S1157A, }}$ AtClo1 was regulated, respectively, by promoters GAL1, TEF1, PGK1 and GAL10.

Saccharomyces cerevisiae were maintained based on their auxotrophy using yeast synthetic complete (SC) 
Table 1 Saccharomyces cerevisiae strain names and introduced genes

\begin{tabular}{|c|c|}
\hline Strain & Genes expressed \\
\hline CON & Empty vectors ${ }^{\mathrm{a}}$ \\
\hline HBYO3 & AtDGAT1 \\
\hline HBY14 & AtDGAT1 Tg/3 $\triangle$ \\
\hline HBY2O & AtDGAT1 Tg/3 $\triangle$ Ald6 SEACS ${ }^{L 641 P}$ \\
\hline $\mathrm{HBY} 31$ & 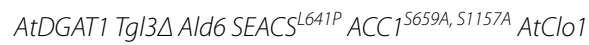 \\
\hline
\end{tabular}

$\Delta$ indicates endogenous gene was knocked out

a pSP-GM2, pIYC04, pESC-leu2d

minimal medium, containing $6.7 \mathrm{~g} / \mathrm{L}$ of yeast nitrogen base, $20 \mathrm{~g} / \mathrm{L}$ glucose as well as a mixture containing appropriate nucleotide bases and amino acids for the various dropout options (SC-Leu, SC-His-Ura, SC-His-LeuUra). All strains were stored in $15 \%$ glycerol at $-80{ }^{\circ} \mathrm{C}$ before being cultured in $5 \mathrm{~mL}$ yeast SC minimal medium and incubated at $30{ }^{\circ} \mathrm{C}, 250 \mathrm{rpm}$, overnight. The culture OD600 nm was diluted to approximately 0.4 into $50 \mathrm{~mL}$ of SC induction medium containing $2 \%(\mathrm{w} / \mathrm{v})$ galactose and $1 \%(\mathrm{w} / \mathrm{v})$ raffinose in $250 \mathrm{~mL}$ flasks. The flasks were capped with aluminum foil and incubated at $30{ }^{\circ} \mathrm{C}, 250$ $\mathrm{rpm}$ until cells were harvested at $72 \mathrm{~h}$ for the following analysis.

\section{GC measurements of total fatty acid content}

Harvested cells were pelleted by centrifugation at 3000 $\mathrm{rpm}$ for $5 \mathrm{~min}$, frozen at $-80{ }^{\circ} \mathrm{C}$ for $\sim 1 \mathrm{~h}$ and subsequently freeze-dried overnight using a FreeZone ${ }^{\circledR} 4.5$ Liter Freeze Dry Systems (Labconco Corporation, USA) to obtain the dry cell weight (DCW) of each culture. Dry cells $(\sim 20 \mathrm{mg})$ were treated with $2 \mathrm{~mL}$ methanol/hydrochloric acid/chloroform (10:1:1, v/v/v) and heated at $90{ }^{\circ} \mathrm{C}$ for $1 \mathrm{~h}$ in sealed test tubes to convert lipids to fatty acid methyl ester (FAME). FAME was washed with $0.9 \%$ $\mathrm{NaCl}$ solution $(1 \mathrm{~mL})$ and extracted with hexane after mixing. FAME samples $(1 \mu \mathrm{L})$ were analyzed by Agilent 7890A gas chromatography with flame ionization detection (GC-FID) as described previously [11].

\section{Lipid body visualization using confocal fluorescence microscope}

Imaging of lipid droplets after Nile red (Sigma-Aldrich, USA) staining of unfixed stationary phase yeast cells was undertaken $72 \mathrm{~h}$ after induction of gene expression $[11,24] .1 \mathrm{~mL}$ of harvested cells were transferred into a $1.5 \mathrm{~mL}$ reaction tube and washed twice with $1 \mathrm{~mL}$ sterile $50 \mathrm{mM}$ Tris- $\mathrm{HCl}$ (pH 7.5). $1 \mu \mathrm{L}$ of Nile Red stock solution was added into the cell suspension to obtain the final concentration of $1 \mu \mathrm{g} / \mathrm{mL}$, gently mixed, incubated for $20 \mathrm{~min}$ at room temperature and centrifuged at $1000 \mathrm{~g}$ for 2 min. $1 \mu \mathrm{L}$ of dense cell suspension was mounted on a standard microscope slide and imaged by a Leica Microsystems SP5 confocal microscope coupled with HCX PL APO $63 \times / 1.4$ OIL CS oil-immersion objective in Monash Micro Imaging, Monash University and the data collected by Leica LAS X (Leica Microsystems, Inc.) microscope control software.

\section{Sample preparation for vibrational spectroscopy-based techniques}

Yeast cells in phosphate buffered saline (PBS) were collected by centrifugation (1000 rpm, $5 \mathrm{~min}$ ) and the pellet resuspended in $500 \mu \mathrm{L}$ of ultrapure water, gently mixed and again centrifuged. This step was repeated 3 times to ensure removal of any residual PBS. The final pellet was resuspended in $500 \mu \mathrm{L}$ of ultrapure water. For each yeast strain, $100 \mu \mathrm{L}$ of the suspension was placed on each of two $\mathrm{CaF}_{2}$ slides and air-dried to obtain a dispersed layer of single cells. From each set of two samples, one was subjected to Raman measurements and the other was mounted on a flat magnetic stainless-steel substrate and designated for AFM-IR measurements. The remaining suspension $(300 \mu \mathrm{L})$ was centrifuged and the pellet was placed directly on the Attenuated Total Reflection (ATR) crystal.

\section{ATR-FTIR measurements}

ATR-FTIR data were recorded using a Bruker Alpha FTIR (Ettlingen, Germany) spectrometer with an ATR sampling device containing a single bounce diamond internal reflection element and equipped with a globar source, $\mathrm{KBr}$ beam splitter and a deuterated triglycine sulfate detector. Spectra were recorded at a resolution of $6 \mathrm{~cm}^{-1}$ in the spectral range of $4000-900 \mathrm{~cm}^{-1}$. For each strain, 3 biological replicates were studied and for each of these, 3 technical replicates were recorded $\left(n_{\text {sin- }}\right.$ gle_strain $\left.=9, n_{\text {total }}=45\right)$. Background spectra were collected directly prior to each measurement (64 scans). After recording the background, $0.5 \mu \mathrm{L}$ of yeast pellet was placed on the crystal and air-dried for approximately 10 min. Each spectrum was recorded using 64 co-added interferograms.

\section{Raman measurements}

Raman measurements were collected using WITec confocal CRM alpha 300 Raman microscope (WITec, Ulm, Germany). The spectrometer was equipped with an air-cooled solid-state laser operating at $532 \mathrm{~nm}$, a CCD detector, cooled to $-60{ }^{\circ} \mathrm{C}$ and 600 grooves $/ \mathrm{mm}$ grating. The laser was coupled to the microscope via an optical fiber with a diameter of $50 \mu \mathrm{m}$. For data collection, a dry Olympus MPLAN $(100 \times / 0.90 \mathrm{NA})$ objective was used. The monochromator of the spectrometer 
was calibrated using Raman scattering line produced by a silicon plate $\left(520.5 \mathrm{~cm}^{-1}\right)$. For each strain, 3 biological replicates were studied and for each of these replicates, 6 individual cells were mapped $\left(n_{\text {single_strain }}=18, n_{\text {to- }}\right.$ tal =90). The size of mapped area was adapted individually, depending on the size of the cell. Data were collected in the spectral range of $3705-0 \mathrm{~cm}^{-1}$, with the spectral resolution of $3 \mathrm{~cm}^{-1}$. The integration time for a single spectrum was $0.1-0.3 \mathrm{~s}$. Laser power was adjusted individually for each sample, not exceeding the range of 5-7 $\mathrm{mW}$. Raman measurements and initial data analysis were performed using WITec software (WITec Plus, Ulm Germany). Raman images were constructed by integration of selected marker bands without any preprocessing. Cluster analysis was carried out after cosmic spike removal (CRR) and background subtraction (polynomial fit, 2nd order). The Raman data were analyzed with $k$-means Clustering (KMC) using the Manhattan distance and Ward's algorithm.

\section{AFM-IR measurements}

AFM-IR measurements were performed with a NanoIR2 system (Anasys Instruments Inc., Santa Barbara, USA). The IR source was an optical parametric oscillator (OPO) laser, producing a $10 \mathrm{~ns}$ pulse at a $1 \mathrm{kHz}$ repetition rate. A silicon cantilever (AppNano, Mountain View, CA 94043, USA) was used with nominal radius of $10 \mathrm{~nm}$ and a nominal spring constant of $0.5 \mathrm{~N} / \mathrm{m}$. The system was purged with $\mathrm{N}_{2}$ to control humidity. For each strain, 3 biological replicates were studied, with 6 single cells investigated for each biological replicate $\left(n_{\text {single_strain }}=18\right.$, $n_{\text {total }}=90$ ). All single spectra were collected in the range of $1800-900 \mathrm{~cm}^{-1}$ with a spectral resolution of $8 \mathrm{~cm}^{-1}$ and IR maps at fixed wavenumber values, to investigate the distribution of selected components (specific wavenumber values are given in Results). Simultaneously to each IR map, the AFM height and deflection images were acquired. The maps were subsequently combined in MatLab (Mathworks, Natick, USA), PLS_toolbox (Eigenvector research, Manson, USA) and analyzed using $k$-means clustering to identify the presence and location of LBs. Following this analysis, single spectra were recorded from cytoplasm and LBs. All presented single spectra were normalized using the Standard Normal Variate (SNV) method and smoothed using the Savitzky-Golay algorithm with 13 smoothing points.

\section{Statistical tests}

Absorbance data obtained for the engineered and control yeast strains were assessed for statistical significance by one way analysis of variance (ANOVA) at $p<0.01$, $\alpha=0.05$; where significant differences were indicated,
Student's $t$ test was applied the post hoc to data for engineered strains compared to control.

\section{Results and discussion \\ Total fatty acid content: correlation between GC-FAME and ATR-FTIR spectroscopy}

The cell lines were firstly characterized using gas chromatography (GC) and ATR-FTIR spectroscopy, to provide an overview of the total fatty acid content (Fig. 1). As can be seen from the GC results (Fig. 1c), the total fatty acid content increased in all modified cell lines compared to control. The most significant increase was observed in the HBY31 cell line carrying alterations in 6 lipid-modifying enzymes, and the increases in lipid were proportional to the number of introduced genes. The same trend was observed in the ATR-FTIR spectra, particularly using the spectral ranges between 1800 and 1500 (Fig. 1d, f) and $3050-2800 \mathrm{~cm}^{-1}$ (Fig. 1e, g).

The first region $\left(1800-1500 \mathrm{~cm}^{-1}\right)$ includes the band at $1745 \mathrm{~cm}^{-1}$ (Fig. 1d, black arrow), attributed to stretching of $\mathrm{C}=\mathrm{O}$ groups of lipids, whereas the second one (2800$3050 \mathrm{~cm}^{-1}$ ) contains a variety of bands originating from stretching of $\mathrm{CH}_{2}$ and $\mathrm{CH}_{3}$ groups in lipid chains [25]. Increase in the intensity of the band at $1745 \mathrm{~cm}^{-1}$ as well as the bands in the high wavenumber region between different cell lines is clearly visible (Fig. $1 \mathrm{~d}-\mathrm{g}$ ) and follows the same trend as an increase in the total fatty acid content assessed from GC (Fig. 1c). Calculation of the ratio of those bands to the amide I band (Fig. 1a, b) enabled the visualization of changes in the lipid to protein ratio [26] in the studied cell lines, as it represents the total fatty acid content in the dried mass of cells. A PLS regression model, built on the basis of 2nd derivatives of ATR-FTIR spectra in the high wavenumber region, enabled the prediction of total fatty acid content (as a percentage of dry cell weight). The correlation between predicted and assessed from GC total fatty acid content for the validation set, together with the parameters of the PLS regression model, is shown in Fig. 1h. This approach provides a fast and straightforward measure of the effectiveness of lipid metabolic engineering strategy. The 2nd derivatives of ATR-FTIR spectra revealed significant variation in the band at $1636 \mathrm{~cm}-1$ (Fig. 1f) assigned to deformation vibration of water, most likely due to intracellular water [27]. No correlation was observed between this and any of the lipid-related bands.

Our initial analysis of metabolically engineered cells undertaken on populations of whole cells addressed the basic question of lipid production between strains and demonstrated the power and rapid analysis of vibrational spectroscopy similar to that previously reported for naturally occurring yeasts by Ami et al. [28]. However, to enable us to address questions relating to subcellular structures and their chemical components and in regard 

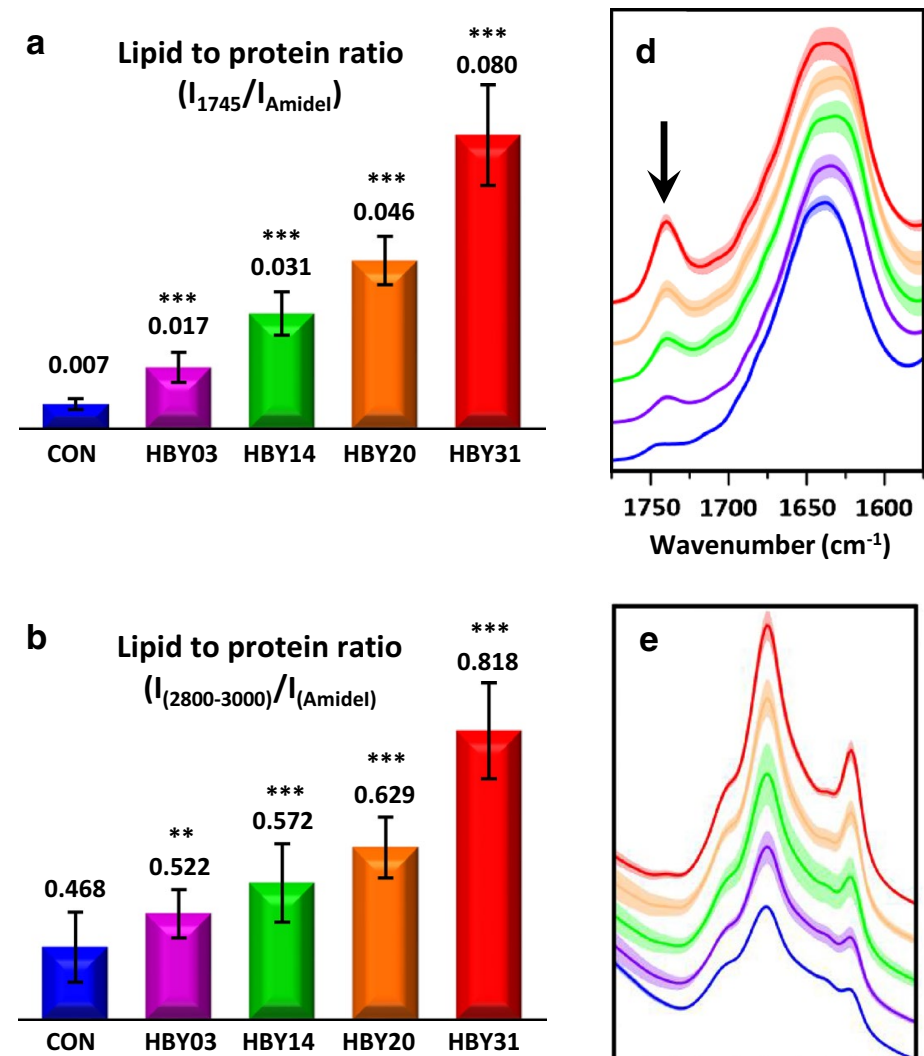

C

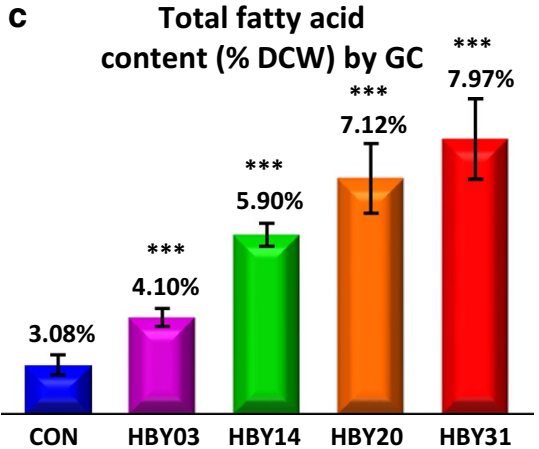

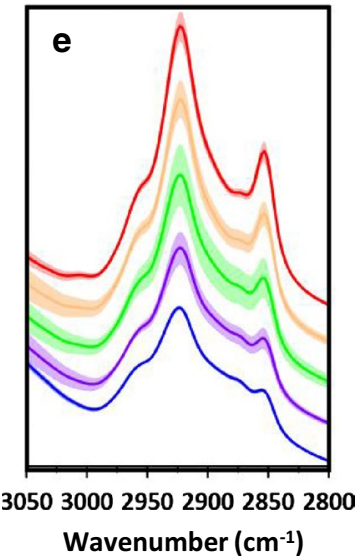
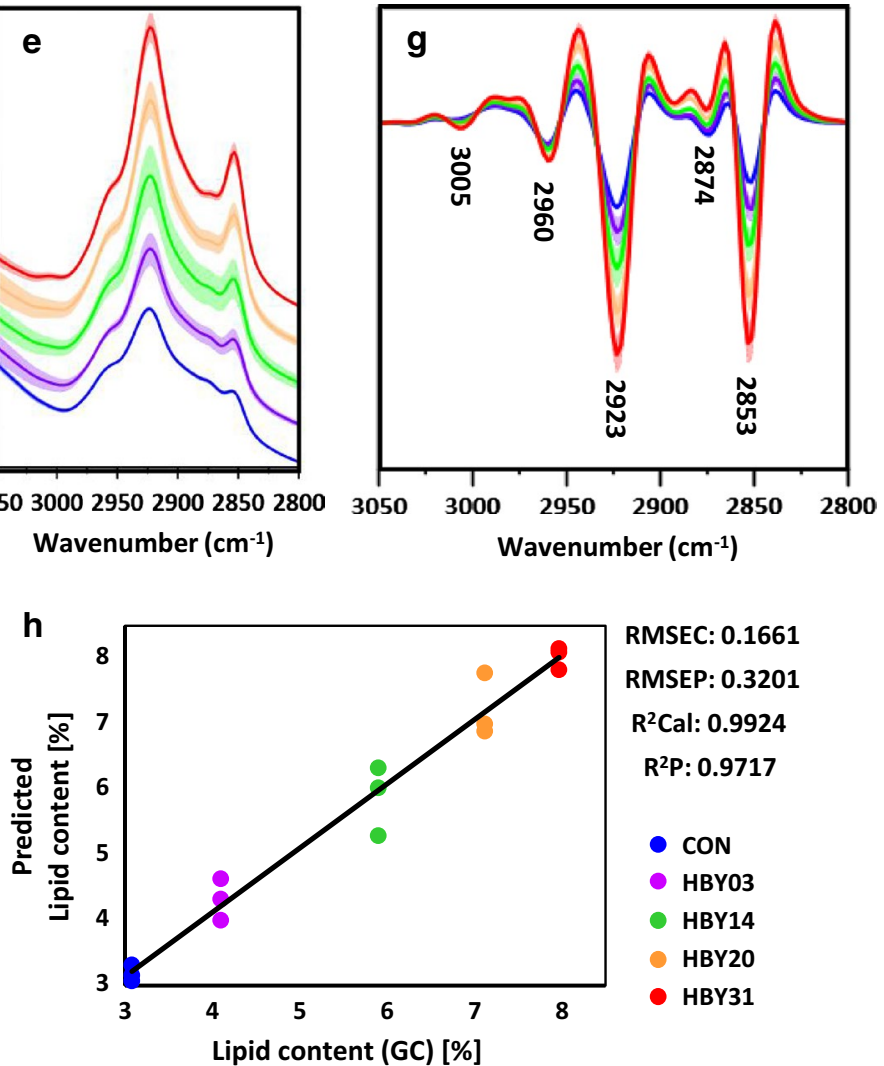

RMSEC: 0.1661

RMSEP: 0.3201

$R^{2}$ Cal: 0.9924

$R^{2} P: 0.9717$

- CON

- HBY03

- HBY14

- HBY20

- HBY31

Fig. 1 Results of the assessment of total fatty acid content obtained via (a, b, d-h) ATR-FTIR and (c) GC for all studies cell lines (CON, HBY03, HBY14, HBY20 and HBY31). (a, b) The ratio of lipid to protein obtained on the basis of ATR-FTIR spectra calculated as the ratio of: $\mathbf{a}$ the band at $1745 \mathrm{~cm}^{-1}$ to the amide I band and $\mathbf{b}$ the bands in high wavenumber region to the amide I band. $\mathbf{c}$ The total fatty acid content obtained for all cell lines through GC. Detailed fatty acid quantification from GC is presented in Additional file 1: Table S1. All bar charts (a-c) show the results obtained together with their standard deviation (SD). d, e Average ATR-FTIR spectra (with SD) of all cell lines and $\mathbf{g}, \mathbf{h}$ their 2 nd derivatives (with SD) used for calculation of ratios presented in $(\mathbf{a})$ and $(\mathbf{b})$ are shown in the range $(\mathbf{d}, \mathbf{f}) 1550-1800 \mathrm{~cm}^{-1}$ and $(\mathbf{e}, \mathbf{g}) 2800-3050 \mathrm{~cm}^{-1}$. Average ATR-FTIR spectra in the whole measured range (3600-600 $\mathrm{cm}^{-1}$ ) are presented in Additional file 1: Fig. S1, S2. h Results of prediction of the total fatty acid content for the validation set by the PLS regression model $\left(2800-3050 \mathrm{~cm}^{-1}\right)$ on the basis of 2 nd derivatives of ATR-FTIR spectra. ${ }^{* *} p<0.01$ vs control and ${ }^{* *} p<0.05$ vs control 
to cell-to-cell variability within metabolic engineered cells required spectroscopic approaches with greater spatial resolution power.

\section{High-resolution CRS visualization and chemical analysis reveals subcellular structures and the impact of metabolic engineering}

To confirm the presence of lipid bodies and estimate their amount, fluorescence imaging of the Nile Red stained cell lines was performed (Fig. 2). The results confirm the presence of LBs in all studied cell lines, with a clear increase in number in the engineered lines compared to control. In addition, amongst the highest engineered line (HBY31), numerous LBs of significantly larger diameter were observed (Fig. 2) but without providing any information about the composition of LBs or other intracellular structures. By comparison high spatial CRS imaging of HBY31 cells visualized LBs via lipid-related bands at $1444 \mathrm{~cm}^{-1}$ (bending mode of $\mathrm{CH}_{2}$ ), $1656 \mathrm{~cm}^{-1}$ (stretching mode of $\mathrm{C}=\mathrm{C}$ ) or $1740 \mathrm{~cm}^{-1}$ (stretching mode $\mathrm{C}=\mathrm{O}$ ) and chemical content through spectra to reveal the presence of saturated (SFA, $1444 \mathrm{~cm}^{-1}$ ), unsaturated fatty acids (UFA, $1656 \mathrm{~cm}^{-1}$ ) as well as triglycerides (TAG, $1740 \mathrm{~cm}^{-1}$ ) (Fig. 3). These confocal spectra ensured the collection of data only from a selected plane of given thickness and thus provide information exclusively about LBs, without interference of lipids from the cytoplasm and cell wall. Cell-to-cell variability in the presence of key metabolites within HBY31 cells is evident within the sample as shown in Fig. 3.

By imaging the cell in different planes, a three-dimensional chemical profile was mapped with the lipid-related bands, attributed to LBs, clearly visible within the cell (Fig. 4a). Via selection of the appropriate plane inside the cell covering a thickness of $\sim 300$ to $400 \mathrm{~nm}$ and spatial identification of LBs within this plane, spectra were obtained from LBs only. Simultaneously, all other cellular structures were studied by integration of marker bands related to their chemical components (Fig. 4b-f) and cluster analysis performed (Fig. 4g) from all spatially localised and averaged spectra obtained from subcellular structures (Fig. 4h). As can be seen, the LBs (Fig. 4b, g, h: red) contained bands attributed to SFAs (1444, $\left.1304 \mathrm{~cm}^{-1}\right)$, UFAs $\left(1656,1268 \mathrm{~cm}^{-1}\right)$, TAGs $\left(1742 \mathrm{~cm}^{-1}\right)$ and phospholipids $\left(1085 \mathrm{~cm}^{-1}\right)$. The CRS spectrum of cell walls (Fig. $4 \mathrm{~g}$, h: dark blue) had a significantly different profile, characteristic of carbohydrates (e.g., 901, $1075 \mathrm{~cm}^{-1}$ ) with some protein contributions $\left(1662 \mathrm{~cm}^{-1}\right)$. Cytoplasm (Fig. 4d, g, h: green) consisted mainly of protein $\left(1662,1342,1314 \mathrm{~cm}^{-1}\right)$, including, e.g., phenylalanine $\left(1007 \mathrm{~cm}^{-1}\right)$ and heme. Within the cytoplasm, areas of high heme content (1590, 1132, $753 \mathrm{~cm}^{-1}$ ) can be identified (Fig. 4e, g, h: brown). Heme (iron protoporphyrin IX) is an essential molecule for yeast; it serves as a prosthetic group in enzymes and proteins especially those involved in transporting oxygen or in oxidation reactions in addition to its many roles in cell signaling, etc. Furthermore, cluster analysis revealed the presence of a large structure (Fig. $4 \mathrm{f}-\mathrm{h}$ : light blue), characterised by marker bands at 1160 and $693 \mathrm{~cm}^{-1}$, attributed to inorganic polyphosphate (PolyP) [22]. PolyP has been reported to accumulate in a variety of organisms including yeast at up to $20 \%$ of dry cell weight [29] and while some biological functions of PolyP are known, its exact physiological role remains unclear. Here, PolyP accumulations were observed only in the most highly engineered cell line (HBY31).

\section{Lipid body characteristics and fatty acid composition of individual engineered yeast cells}

To investigate the variability in presence of LBs, their size and composition within the same metabolic engineered line and between the cell lines, CRS imaging was applied to a total of 90 cells drawn equally from all strains. The distribution of selected components (organic matter, lipids and heme) from a subgroup of these cells is shown in Fig. 5 together with the total number of observed LBs in each cell line and their average diameters. In the case of the control, HBY03 and HBY14 strains show single LBs (per cell) with a diameter of $\sim 1 \mu \mathrm{m}$, or lacking obvious LBs, with a minor increase in the number of LBs in the engineered lines compared to control. A significant increase in the number of LBs in cells of HBY2O and HBY31 was observed, with HBY20 having the highest among all strains and with LBs present in every cell.

Interestingly, LBs in HBY20 were only slightly increased in size (diameter $1.21 \pm 0.40 \mu \mathrm{m}$ ) compared to control (diameter $0.87 \pm 0.23 \mu \mathrm{m}$ ) (Fig. 5). For HBY31 strain, LBs were observed in all cells, however, in two types of arrangements. Some cells, similarly to HBY20, contained multiple LBs with a diameter of approximately $1 \mu \mathrm{m}$, but others contained a single large LB which filled the cell almost entirely (Fig. 5, HBY31 Panel G). This resulted in fewer but overall significantly larger LBs in HBY31 $(2.14 \pm 1.08 \mu \mathrm{m})$ compared to any other strain. HBY31 was the only strain assessed in this study that expressed caleosin (Table 1), a lipid droplet stabilizing protein originating from plants which may account for the larger LBs observed here.

For a detailed investigation of LB composition, average spectra of each LB from each cell line (calculated from spectra of single LBs) were generated and variability measured (Fig. 6). Spectra of LBs contain several bands in the fingerprint region that are attributed to UFAs $\left(1656,1268 \mathrm{~cm}^{-1}\right)$ and SFAs $\left(1444,1304 \mathrm{~cm}^{-1}\right)$ with the ratio of intensities of $\mathrm{I}_{1656} / \mathrm{I}_{1444}$ being among the best 

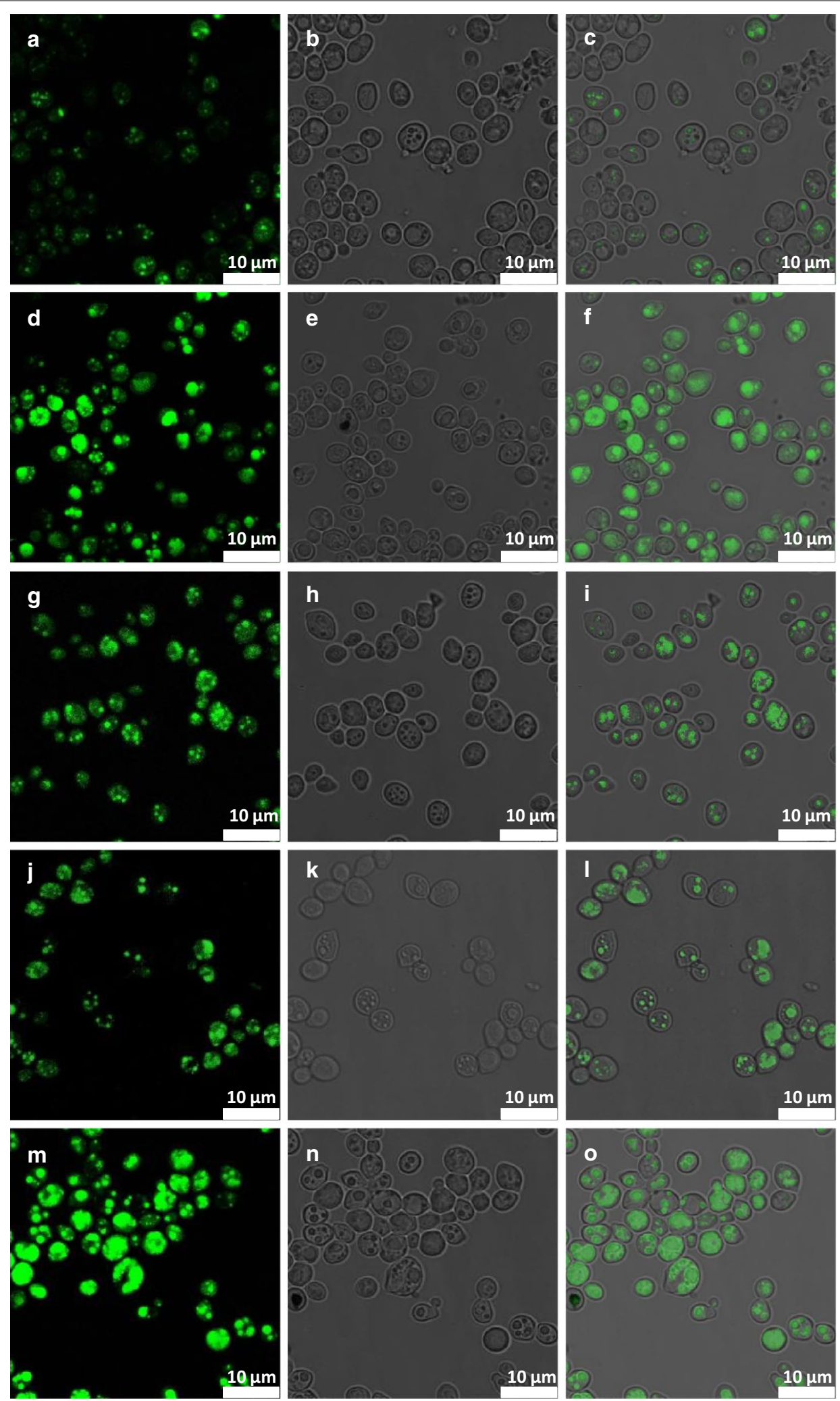

Fig. 2 Images showing: $(\mathbf{a}, \mathbf{d}, \mathbf{g}, \mathbf{j}, \mathbf{m})$ fluorescence results, $(\mathbf{b}, \mathbf{e}, \mathbf{h}, \mathbf{k}, \mathbf{n})$ visible image in transmission and $(\mathbf{c}, \mathbf{f}, \mathbf{i}, \mathbf{l}, \mathbf{o})$ overlay of visible and fluorescence image obtained for the: (a-c) CON, (d-f) HBY03, (g-i) HBY14, (j-I) HBY20 and (m-o) HBY31 yeast cell line at $72 \mathrm{~h}$ culturing. The scale bar is presented at the bottom right corner of each image 


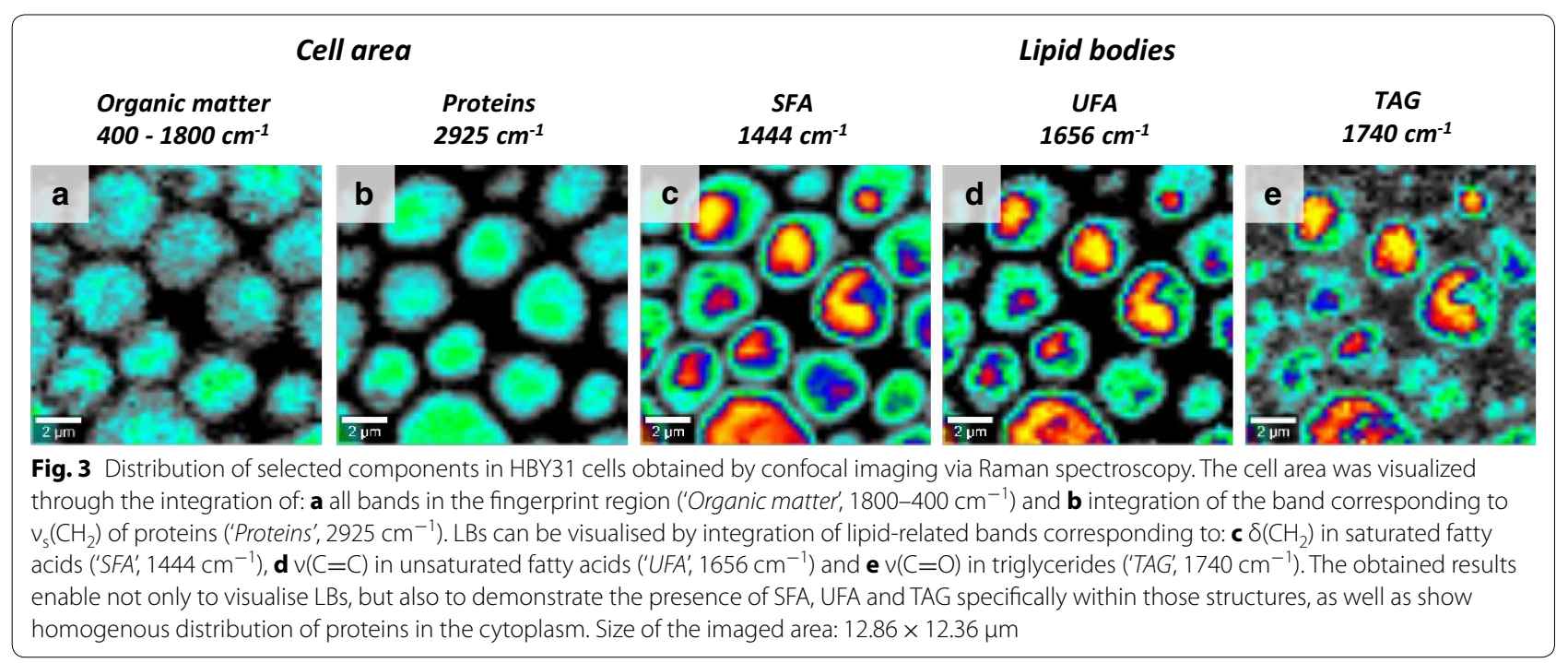

indicators of fatty acid unsaturation [30]. An alternative approach for measuring fatty acid unsaturation by comparing intensities at 3012 and $2855 \mathrm{~cm}^{-1}$ is much less sensitive to unsaturation changes. No significant difference was found in the degree of unsaturation in LBs from HBY03 and HBY14 strains compared to control, but a significant decrease in unsaturation of LBs was observed for HBY31 $(0.32 \pm 0.05)$, and to a lesser extent HBY20 (Fig. 6). The observed decrease in fatty acid unsaturation was unlikely to be due to the larger size of LBs in HBY31 as the reduction was also observed in HBY20 for which no change in LB size was noted. This result indicated a shift to higher saturated fatty acid content in LBs in the more engineered strains measured via spectroscopy and the result was supported by GC analysis of fatty acid composition (Additional file 1: Table S1). It should be noted that CRS analysis was specifically measured in LB whereas GC analysis encompassed the whole cell fatty acid composition.

The shift to higher saturated fatty acid content in the most highly engineered strains was not only due to the specificity of the introduced acyltransferase, DGAT1, for saturated fatty acids as the AtDGAT1 gene was expressed in all strains but maybe also due to limited quantities of unsaturated fatty acids available for conversion to triacylglycerol. $\Delta 9$-Unsaturated fatty acids are essential components of plasma membranes of yeast [31] and may be preferentially directed to these locations potentially resulting in more saturated fatty acids directed to triacylglycerol production through the enhanced expression of diacylglycerol acyltransferases.

\section{Comprehensive mapping of cellular constituents by AFM-} IR

To obtain a comprehensive measure of biochemical changes in cells resulting from induced genetic modifications, further analysis of the composition of cytoplasm and, in particular, carbohydrate content was required. The ability to simultaneously study the composition of intracellular structures without the need to isolate them is an advantage of high spatial imaging via vibrational spectroscopy. However, as described earlier, the cytoplasm of engineered yeast contains significant and varying amounts of heme in the form of clusters and dispersed within the cytoplasm (Fig. 4e, g, h-Brown). The raised and variable Raman background due to heme content effectively conceals less intense signals such as carbohydrate-related bands excited at lower wavelengths and therefore, IR-based spectroscopy is more useful to investigate carbohydrate components at high spatial resolution. However, as the spatial resolution of conventional IR imaging does not exceed $\sim 5 \mu \mathrm{m}$, a more sophisticated approach based on AFM-IR imaging was applied in our case. In AFM-IR the phenomenon of IR absorption is not measured directly, but by measuring the thermal expansion of the sample resulting from application. Therefore, the spatial resolution of imaging with the use of AFM-IR is no longer limited to $\sim 5 \mu \mathrm{m}$, but can be significantly smaller $(\sim 100 \mathrm{~nm})$, enabling the measurement of spectra representing explicitly the composition of subcellular structures. AFM-IR mapping with selected bands corresponding to proteins, lipids and carbohydrates was performed on 6 replicate cells for each strain and the images were combined and analysed via cluster analysis to confirm presence (or absence) of LBs and determine their precise location within the 


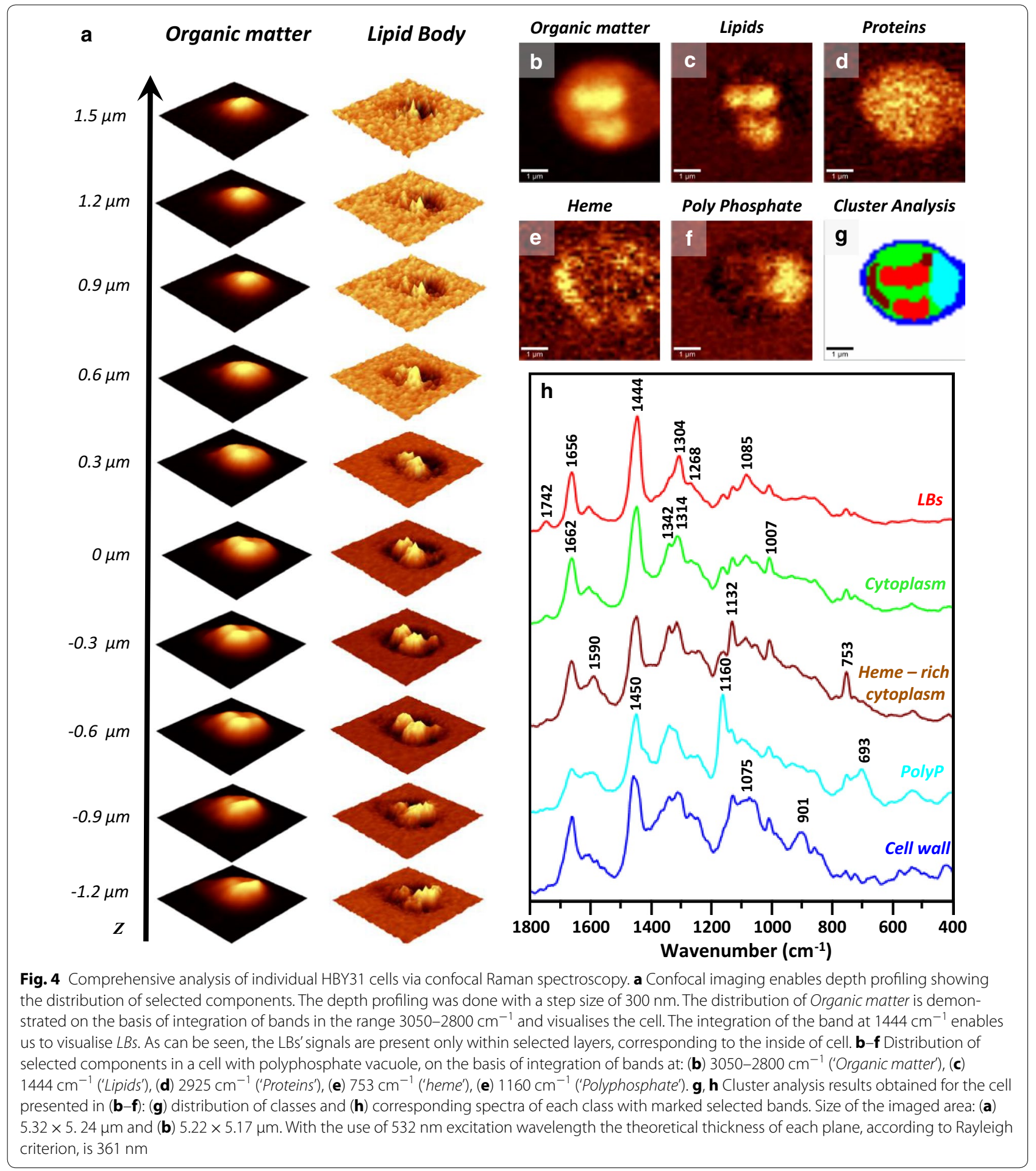

cell. Subsequently, single spectra (5-10) were collected from areas corresponding to LBs and cytoplasm. A high prevalence of LBs in the HBY31 and HBY20 strains were observed, in agreement with results shown previously by other techniques applied in this study.
Focussing on HBY31 strain, Fig. 7a-d shows the AFM height profile recorded simultaneously with each AFMIR map and the relatively even thickness of the cell (approximately $3 \mu \mathrm{m}$ ). The presence of LBs was revealed through imaging of the absorbance at $1740 \mathrm{~cm}^{-1}$ 


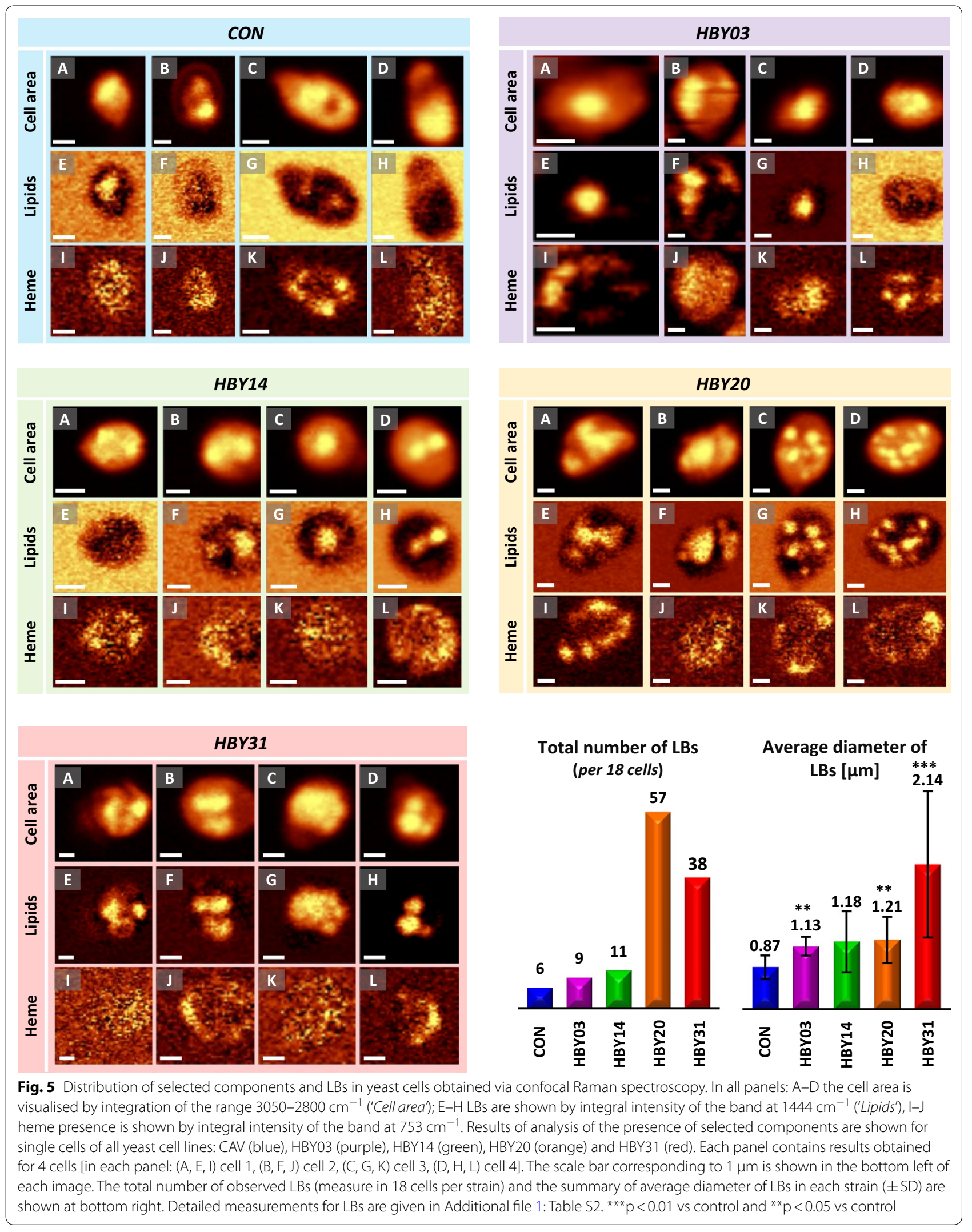



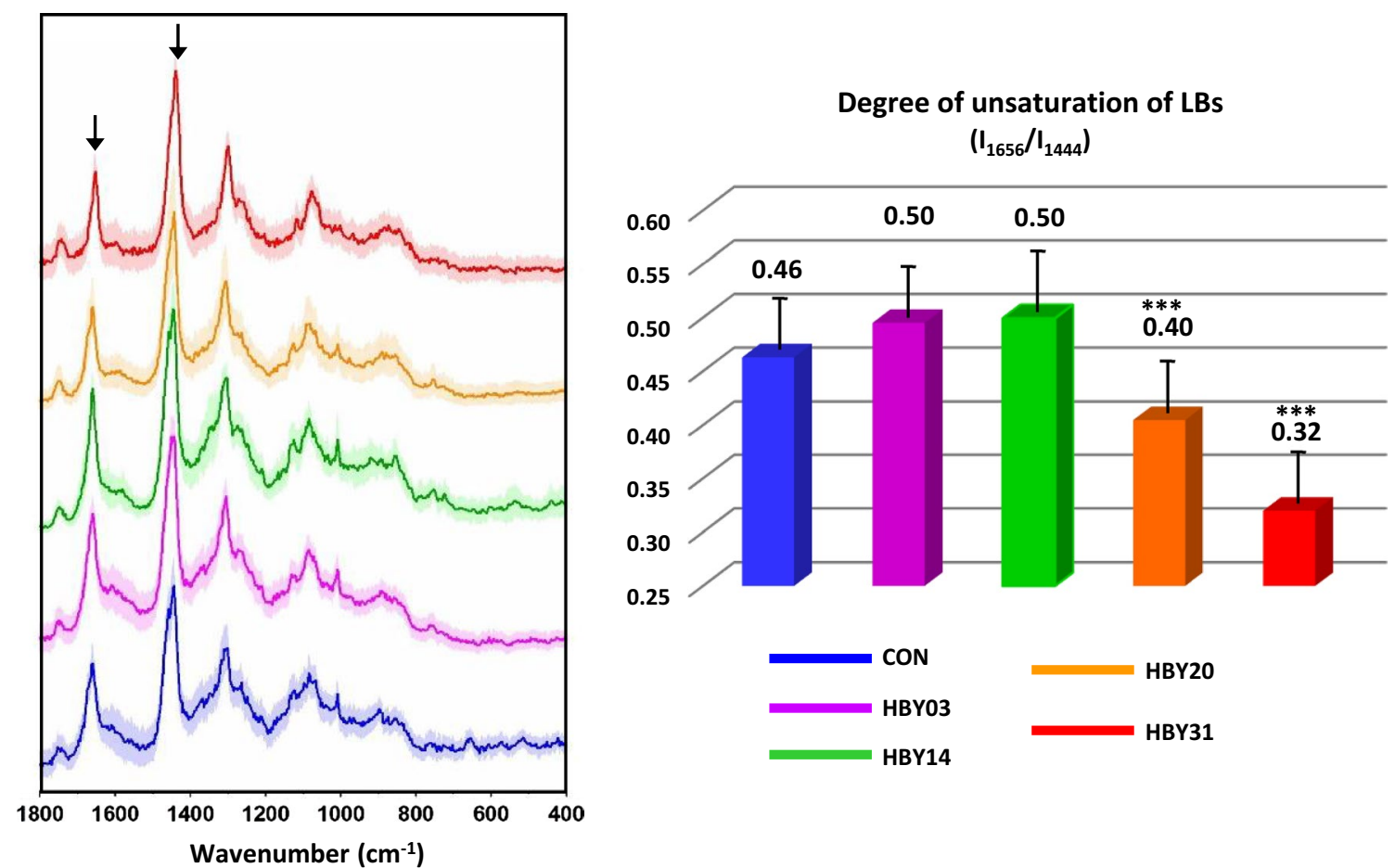

Fig. 6 Assessment of the degree of unsaturation of LBs in engineered yeast strains. (Left) Average Raman spectra from all measured LBs (per cell line) together with their standard deviation, in the range $1800-400 \mathrm{~cm}^{-1}$. Black arrows mark the bands used for calculation of the degree of unsaturation. (Right) Degree of unsaturation of LBs calculated as the ratio of the band at $1656 \mathrm{~cm}^{-1}(\mathrm{C}=\mathrm{C})$ to the band at $1444 \mathrm{~cm}^{-1}\left(\mathrm{CH}_{2}\right)($ average value $\pm S D) .{ }^{* *} p<0.01$ vs control

(stretching of $\mathrm{C}=\mathrm{O}$ from lipids) (Fig. $7 \mathrm{f}$ ) and $1264 \mathrm{~cm}^{-1}$ (deformation of $\mathrm{CH}_{2}$ of lipids) (Fig. $7 \mathrm{~g}$ ). The protein (Fig. 7e) and carbohydrate (Fig. 7h) distribution in the cells were homogenous, as these signals originate from both cytoplasm and cell wall, with the latter being of even thickness across the cell. A comparison between AFMIR spectra of LB (Fig. 7i, red) and cytoplasm (Fig. 7i, black) of the same HBY31 cell showed large differences, the LB spectrum was dominated by lipid-related signals (1740, 1464, $1080 \mathrm{~cm}^{-1}$ ) and an intense phospholipid band $\left(1080 \mathrm{~cm}^{-1}\right.$, stretching of $\left.\mathrm{PO}_{2}\right)$ originating from the LB membrane. The spectra of the HBY31 cytoplasm (Fig. 7i, black) has higher protein $\left(1656 \mathrm{~cm}^{-1}\right)$ to lipid $\left(1740 \mathrm{~cm}^{-1}\right)$ ratio compared with the LB spectra (Fig. $7 \mathrm{i}$, red). A comparison of 2nd derivatives of AFM-IR spectra of cytoplasm from both HBY31 (Fig. 7j, black) and control (Fig. 7j, blue) showed interesting differences: although the HBY31 cytoplasm shows lower intensity due to lipids than LB from the same cell, it reveals a higher lipid content in the cytoplasm, compared to the control cell (Fig. 7j, blue). In terms of metabolic engineering, the measurement of significant quantities of lipid in the cytoplasm suggests the lipid accumulation and sequestration strategies for HBY31 cells are out of balance with production, and lipid storage capacity of LBs in this strain has been overwhelmed. In addition, a band of significant intensity at $1044 \mathrm{~cm}^{-1}$ in control cells (Fig. 7j, blue) attributed to carbohydrate, was absent in the cytoplasm of HBY31 (Fig. 7j, black), suggesting an acute reduction of the carbohydrates in favour of enrichment of lipids in the cytoplasm of HBY31.

\section{AFM-IR reveals cell-to-cell variability in concentrations of key metabolites}

The AFM-IR spectra obtained from individual cells in all strains were analysed with respect to the relationship between carbohydrate and lipid content, and variance between samples. The average spectra for cytoplasm for each strain are shown in Fig. 8 together with the variance within the strain. The lipid content in the cytoplasm (e.g., $1740 \mathrm{~cm}^{-1}$ ) undoubtedly increases, from low levels in control cells, through higher amounts for HBY03 and HBY14, to very high concentrations for HBY20 and HBY31 cell lines (Fig. 8a, c marked with a star), and the intra-strain variability in cytoplasm lipid increases. Concurrent with the increase of the intensity of lipid-related bands with more engineered strains, was a decrease in intensity of the bands in the range $1100-900 \mathrm{~cm}^{-1}$ 


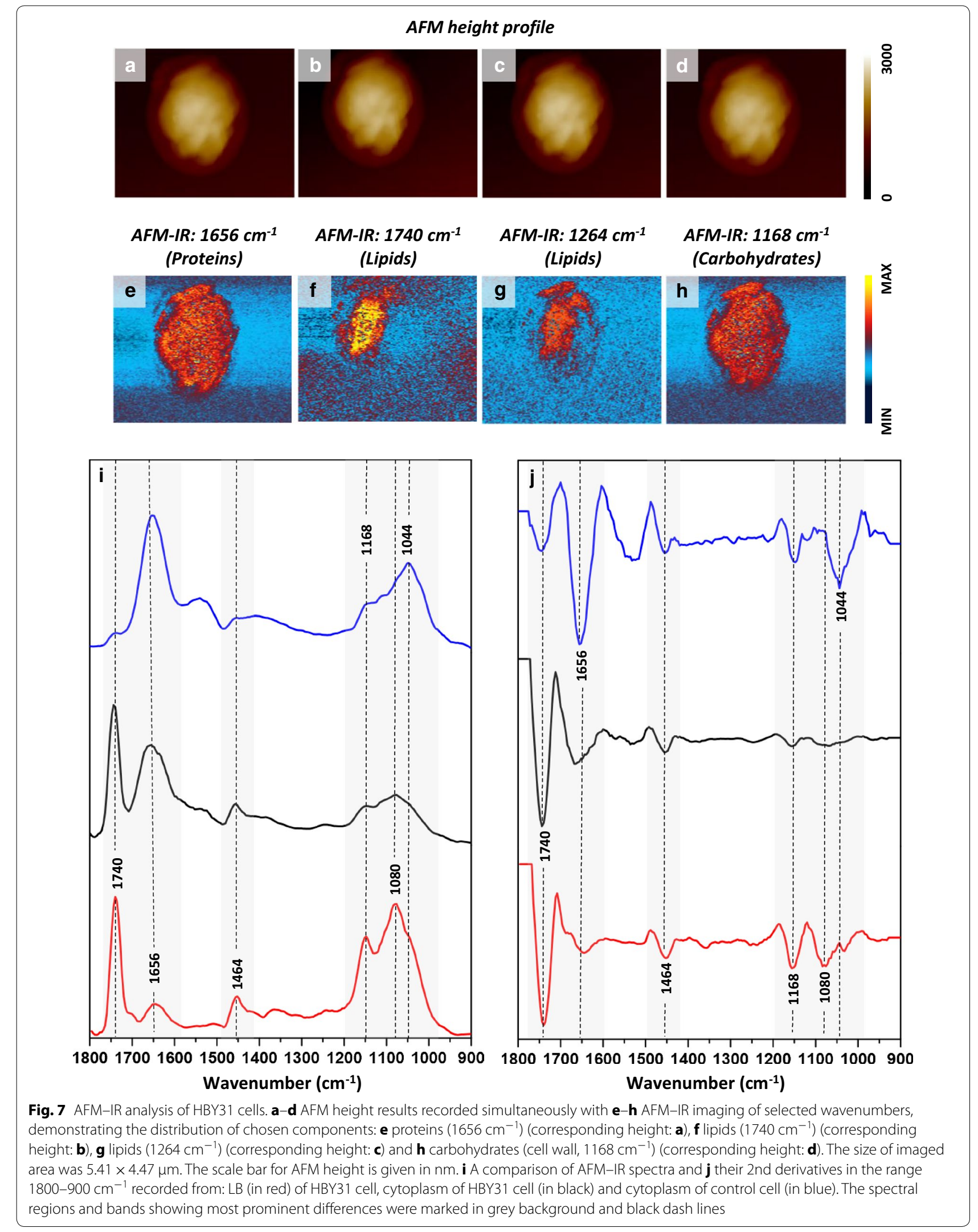



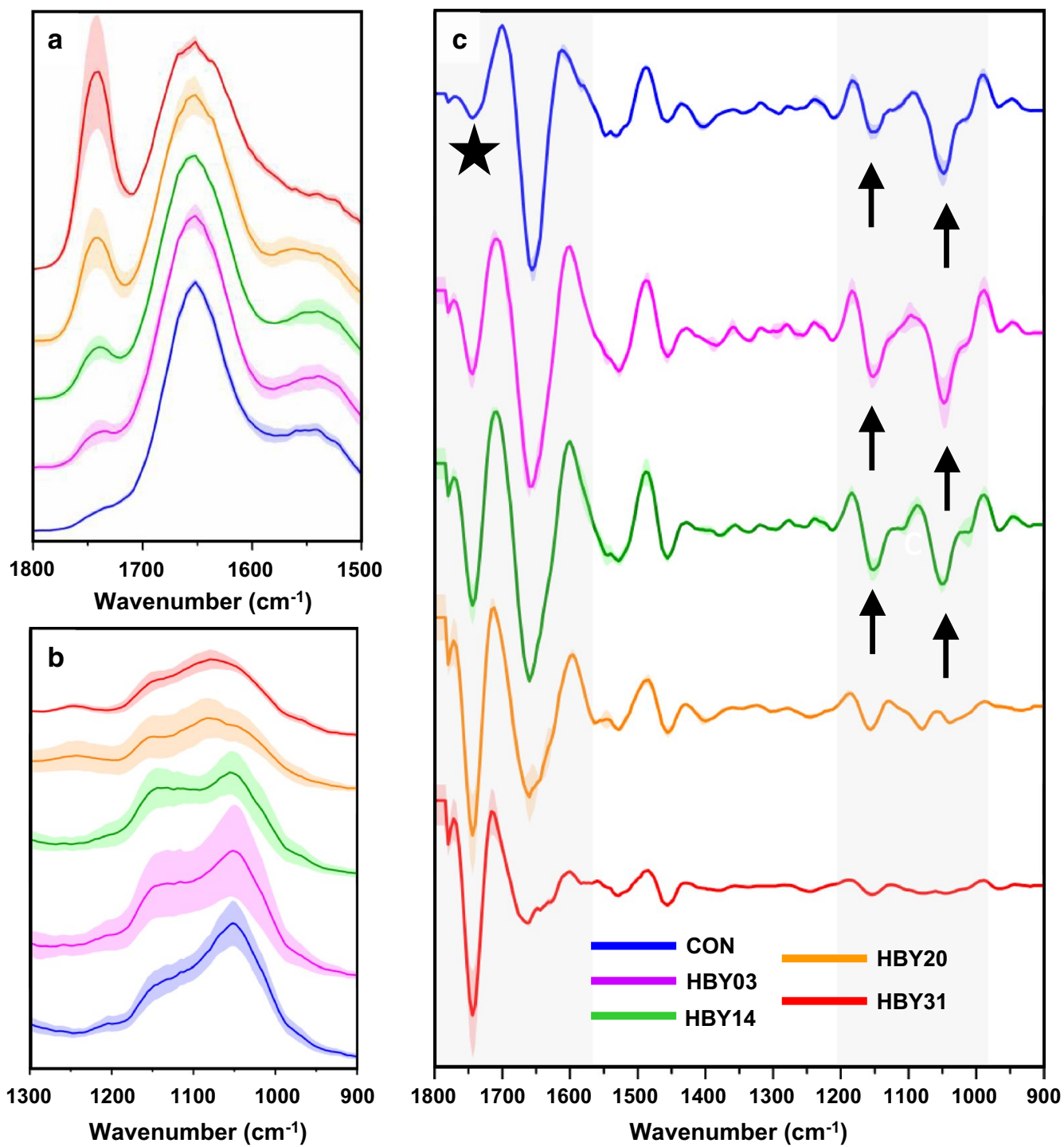

Fig. 8 Averaged AFM-IR spectra of cytoplasm of engineered yeast cells in the range (a) $1800-1500 \mathrm{~cm}^{-1}$ and (b) $1300-900 \mathrm{~cm}^{-1}$ together with c their 2nd derivative in the range $1800-900 \mathrm{~cm}^{-1}$. Each presented average spectrum was obtained by averaging 30 single spectra collected for different cells (of the same strain) and is shown with SD. Each single spectrum in this study was collected from cytoplasm, after localization of the LB (or confirmation of its absence) through AFM-IR imaging. Grey area, black star and black arrows mark the most prominent differences

(Fig. 8b). A detailed comparison of 2nd derivative spectra revealed the contribution of different components in this region: for control, HBY03 and HBY14, the carbohydraterelated band at $1044 \mathrm{~cm}^{-1}$ was clearly present (Fig. 8c, black arrows) and of significant intensity. For cytoplasm of HBY20, the carbohydrate band was just visible but substantially lower and the emergence of the phospholipid band at $1080 \mathrm{~cm}^{-1}$ was evident (Fig. 8b). As discussed previously, the cytoplasm of HBY31 cells had almost no intensity at $1044 \mathrm{~cm}^{-1}$ whereas the band at $1080 \mathrm{~cm}^{-1}$ became more prominent (Fig. 8b). Altogether, the AFMIR results demonstrate the increase in lipid content and a simultaneous decrease in the carbohydrate content in the cytoplasm, from high carbohydrate and low lipid in control cells to the reversed ratio in the HBY31 strain.

Vibrational spectroscopy analysis has previously been shown to be highly informative for measurement and location of target metabolites such as lipids in cultured cells of wild-type microalgae and yeast [17, 19, 32-40] and in selected studies that include heterologous expression of lipid stabilizing proteins [41]. Our study is the first to our knowledge where the effects of the sequential introduction of a complex metabolic engineering pathway into an organism have been measured using 
vibrational spectroscopy techniques and have revealed valuable insights into areas for further improvement.

\section{Conclusions}

Here, we have used vibrational spectroscopy techniques to measure the impact of metabolic engineering approaches for high lipid production in yeast at both the single cell level and averaged and detailed information about the chemical composition of subcellular structures. The ratio of lipid-related bands (e.g., $1740 \mathrm{~cm}^{-1}$ ) to amide I was demonstrated to be useful as a quick marker of the total lipid content. An established PLS regression model allowed the successful prediction of total fatty acid content from the ATR-FTIR spectrum, demonstrating the ability of this technique to provide the same information as GC, but within few minutes and without the need for complex sample preparation. Fluorescence imaging enabled us to visualise LBs, confirming their highest prevalence and size in the HBY31 strain. Furthermore, two high spatial imaging techniques based on vibrational spectroscopies were applied (CRS, AFM-IR) and demonstrated substantial changes in intracellular composition with more complex metabolic engineering. CRS imaging provided an insight into LBs composition, revealing a significant decrease in the degree of unsaturation of lipids in the most highly engineered strain-HBY31. In addition, CRS imaging demonstrated significantly increased LB number in HBY20 and HBY31 strains together with a dramatic increase in LB size for HBY31 that expressed the LB stabilizing protein, caleosin. Finally, the AFM-IR imaging demonstrated large changes in the composition of cytoplasm between strains. A decrease in carbohydrate content with concurrent increase in lipid content of cytoplasm was observed, progressing from control through to HBY31 strains. The high concentration of lipid in the cytoplasm of HBY31, in particular, suggests lipid production rate in these engineered yeast is exceeding the rate of lipid sequestration in LBs which could lead to lipotoxicity. Additionally, the shift towards higher saturated fatty acids levels in stored lipids suggests a reduction in the availability of unsaturated fatty acids for TAG formation in the cells. Altogether, our results have demonstrated an increase in the total lipid content resulting from genetic modifications, with the multigene modification approach (Ald6, SEACS ${ }^{L 641 P}, A C C 1^{\text {S659A, S1157A }}$ AtDGAT1, AtClo1 and $T g l 3 \triangle$ ) in the HBY31 cell line being most effective. The vibrational spectroscopy approach allowed the simultaneous measurement of intra-strain variability in metabolite production and impact on cellular structures from metabolic engineering.

\section{Additional file}

Additional file 1. Supplementary Information.

\section{Abbreviations}

ACC: acetyl-CoA carboxylase; ACS: acetyl-CoA synthetase; AFM-IR: atomic force microscopy-infrared spectroscopy; ALD: aldehyde dehydrogenase; AtClo1: caleosin from Arabidopsis thaliana; ATR-FTIR: attenuated total reflection-Fourier transform infrared spectroscopy; CRS: confocal Raman spectroscopy; DCW: dry cell weight; DGAT1: diacylglycerol acyltransferase; FA: fatty acid; FAME: fatty acid methyl ester; GC: gas chromatography; GC-FID: gas chromatography with flame ionization detector; IR: infrared (spectroscopy); LB: lipid body; PBS: phosphate buffer solution; PLS: partial least square; PolyP: inorganic polyphosphate; RS: Raman spectroscopy; SFA: saturated fatty acid; SNV: standard normal variate; TAG: triacylglycerol; Tgl3: triacylglycerol lipase 3; UFA: unsaturated fatty acid.

\section{Authors' contributions}

KK collected ATR, Raman, AFM-IR, performed the data analysis, prepared the figures and drafted the manuscript. HP cultured the yeast cell lines, collected and analysed GC and fluorescence data and contributed to writing and correcting the manuscript. BR contributed to the experimental planning and corrected the manuscript. VH contributed to the design of the experiment, data interpretation and contributed to the writing of the manuscript. All authors read and approved the final manuscript.

\section{Author details}

${ }^{1}$ Centre for Biospectroscopy, School of Chemistry, Monash University, Clayton Campus, Clayton, VIC 3800, Australia. ${ }^{2}$ Department of Chemical Engineering, Monash University, Clayton Campus, Clayton, VIC 3800, Australia.

\section{Acknowledgements}

We thank Mr. Finlay Shanks for technical support of the instrumentals and are grateful to Ms. Yanqin Xu and Prof. Hongyuan Yang for the gift of the strains BY4741 and BY4741 Tg/3 $\triangle$.

\section{Competing interests}

The authors declare that they have no competing interests.

\section{Availability of data and materials}

The datasets used and/or analysed during the current study are available from the corresponding author on reasonable request.

\section{Consent for publication}

Not applicable.

\section{Ethics approval and consent to participate}

Not applicable.

\section{Funding}

We acknowledge the financial support from ARC linkage Project (LE160100185) and Monash University for the graduate and international postgraduate research scholarships awarded to HP. B.R.W. is supported by an Australian Research Council (ARC) Future Fellowship Grant FT120100926.

\section{Publisher's Note}

Springer Nature remains neutral with regard to jurisdictional claims in published maps and institutional affiliations.

Received: 19 February 2018 Accepted: 4 April 2018

Published online: 10 April 2018

\section{References}

1. Liang MH, Jiang JG. Advancing oleaginous microorganisms to produce lipid via metabolic engineering technology. Prog Lipid Res. 2013;52(4):395-408.

2. Lennen RM, Pfleger BF. Microbial production of fatty acid-derived fuels and chemicals. Curr Opin Biotechnol. 2013;24(6):1044-53.

3. Lamers D, van Biezen N, Martens D, Peters L, van de Zilver E, Jacobs-van Dreumel N, Wijffels RH, Lokman C. Selection of oleaginous yeasts for fatty acid production. BMC Biotechnol. 2016;16(1):45. 
4. Liu Y, Zhang C, Shen X, Zhang X, Cichello S, Guan H, Liu P. Microorganism lipid droplets and biofuel development. BMB Rep. 2013;46(12):575-81.

5. Zhou YJ, Buijs NA, Zhu Z, Qin J, Siewers V, Nielsen J. Production of fatty acid-derived oleochemicals and biofuels by synthetic yeast cell factories. Nat Commun. 2016;7:11709.

6. Sitepu IR, Garay LA, Sestric R, Levin D, Block DE, German JB, Boundy-Mills $\mathrm{KL}$. Oleaginous yeasts for biodiesel: current and future trends in biology and production. Biotechnol Adv. 2014;32(7):1336-60

7. Friedlander J, Tsakraklides V, Kamineni A, Greenhagen EH, Consiglio AL, MacEwen K, Crabtree DV, Afshar J, Nugent RL, Hamilton MA, et al. Engineering of a high lipid producing Yarrowia lipolytica strain. Biotechnol Biofuels. 2016;9(1):77.

8. Chen Y, Daviet L, Schalk M, Siewers V, Nielsen J. Establishing a platform cell factory through engineering of yeast acetyl-CoA metabolism. Metab Eng. 2013;15(Supplement C):48-54.

9. Ruenwai R, Cheevadhanarak S, Laoteng K. Overexpression of acetylCoA carboxylase gene of Mucor rouxii enhanced fatty acid content in Hansenula polymorpha. Mol Biotechnol. 2009:42(3):327-32.

10. Tai M, Stephanopoulos G. Engineering the push and pull of lipid biosynthesis in oleaginous yeast Yarrowia lipolytica for biofuel production. Metab Eng. 2013;15(Supplement C):1-9.

11. Peng H, Moghaddam L, Brinin A, Williams B, Mundree S, Haritos VS. Functional assessment of plant and microalgal lipid pathway genes in yeast to enhance microbial industrial oil production. Biotechnol Appl Biochem 2017:1-7. https://doi.org/10.1002/bab.1573.

12. Froissard M, D'Andrea S, Boulard C, Chardot T. Heterologous expression of AtClo1, a plant oil body protein, induces lipid accumulation in yeast. FEMS Yeast Res. 2009;9(3):428-38.

13. Dulermo T, Nicaud J-M. Involvement of the G3P shuttle and $\beta$-oxidation pathway in the control of TAG synthesis and lipid accumulation in Yarrowia lipolytica. Metab Eng. 2011;13(5):482-91.

14. Martins BM, Locke JC. Microbial individuality: how single-cell heterogeneity enables population level strategies. Curr Opin Microbiol. 2015;24:104-12.

15. Fritzsch FS, Dusny C, Frick O, Schmid A. Single-cell analysis in biotechnology, systems biology, and biocatalysis. Ann Rev Chem Biomol Eng. 2012;3:129-55.

16. Radulovic M, Knittelfelder O, Cristobal-Sarramian A, Kolb D, Wolinski H, Kohlwein SD. The emergence of lipid droplets in yeast: current status and experimental approaches. Curr Genet. 2013;59(4):231-42.

17. Smith R, Wright KL, Ashton L. Raman spectroscopy: an evolving technique for live cell studies. Analyst. 2016;141(12):3590-600.

18. Heraud P, Marzec KM, Zhang QH, Yuen WS, Carroll J, Wood BR. Labelfree in vivo Raman microspectroscopic imaging of the macromolecular architecture of oocytes. Sci Rep. 2017;7(1):8945

19. Kochan K, Kus E, Filipek A, Szafranska K, Chlopicki S, Baranska M. Labelfree spectroscopic characterization of live liver sinusoidal endothelial cells (LSECs) isolated from the murine liver. Analyst. 2017;142(8):1308-19.

20. Perez-Guaita D, Kochan K, Martin M, Andrew DW, Heraud P, Richards JS, Wood BR. Multimodal vibrational imaging of cells. Vib Spectrosc. 2017;91(Supplement C):46-58.

21. Shao Y, Fang H, Zhou H, Wang Q, Zhu Y, He Y. Detection and imaging of lipids of Scenedesmus obliquus based on confocal Raman microspectroscopy. Biotechnol Biofuels. 2017;10:300.

22. Noothalapati H, Sasaki T, Kaino T, Kawamukai M, Ando M, Hamaguchi HO, Yamamoto T. Label-free chemical imaging of fungal spore walls by Raman microscopy and multivariate curve resolution analysis. Sci Rep. 2016;6:27789.

23. Peng; $\mathrm{H}, \mathrm{He}$; L, Haritos VS: Impact of yeast lipid pathway engineering and bioprocess strategy on cellular physiology and lipid content. J Ind Microbiol Biotechnol. 2018 (under review).
24. Wolinski H, Kohlwein SD. Microscopic and spectroscopic techniques to investigate lipid droplet formation and turnover in yeast. Membrane Trafficking: Second Edition; 2015. p. 289-305.

25. Cavagna M, Dell'Anna R, Monti F, Rossi F, Torriani S. Use of ATR-FTIR microspectroscopy to monitor autolysis of Saccharomyces cerevisiae cells in a base wine. J Agric Food Chem. 2010;58(1):39-45

26. Kochan K, Maslak E, Chlopicki S, Baranska M. FT-IR imaging for quantitative determination of liver fat content in non-alcoholic fatty liver. Analyst. 2015;140(15):4997-5002.

27. Mojet BL, Ebbesen SD, Lefferts L. Light at the interface: the potential of attenuated total reflection infrared spectroscopy for understanding heterogeneous catalysis in water. Chem Soc Rev. 2010;39(12):4643-55.

28. Ami D, Posteri R, Mereghetti P, Porro D, Doglia SM, Branduardi P. Fourier transform infrared spectroscopy as a method to study lipid accumulation in oleaginous yeasts. Biotechnol Biofuels. 2014;7(1):12.

29. McGrath JW, Quinn JP. Intracellular accumulation of polyphosphate by the yeast Candida humicola G-1 in response to acid pH. Appl Environ Microbiol. 2000:66(9):4068-73

30. Kochan K, Maslak E, Krafft C, Kostogrys R, Chlopicki S, Baranska M. Raman spectroscopy analysis of lipid droplets content, distribution and saturation level in non-alcoholic fatty liver disease in mice. J Biophotonics. 2015:8(7):597-609.

31. Stukey JE, MCDonough VM, Martin CE. The OLE1 gene of Saccharomyces cerevisiae encodes the delta 9 fatty acid desaturase and can be functionally replaced by the rat stearoyl-CoA desaturase gene. J Biol Chem. 1990;265(33):20144-9.

32. Shao YN, Fang H, Zhou H, Wang Q, Zhu YM, He Y. Detection and imaging of lipids of Scenedesmus obliquus based on confocal Raman microspectroscopy. Biotechnol Biofuels. 2017:10:300.

33. Wang TT, Ji YT, Wang Y, Jia J, Li J, Huang S, Han DX, Hu Q, Huang WE, Xu J. Quantitative dynamics of triacylglycerol accumulation in microalgae populations at single-cell resolution revealed by Raman microspectroscopy. Biotechnol Biofuels. 2014:7:58

34. Chiu LD, Ho SH, Shimada R, Ren NQ, Ozawa T. Rapid in vivo lipid/carbohydrate quantification of single microalgal cell by Raman spectral imaging to reveal salinity-induced starch-to-lipid shift. Biotechnol Biofuels. 2017;10:9.

35. He YH, Zhang P, Huang S, Wang TT, Ji YT, Xu J. Label-free, simultaneous quantification of starch, protein and triacylglycerol in single microalgal cells. Biotechnol Biofuels. 2017:10:275.

36. Noothalapati H, Sasaki T, Kaino T, Kawamukai M, Ando M, Hamaguchi $\mathrm{H}$, Yamamoto T. Label-free chemical imaging of fungal spore walls by Raman microscopy and multivariate curve resolution analysis. Sci Rep. 2016;6:27789.

37. Huang $\mathrm{CK}$, Hamaguchi $H$, Shigeto $S$. In vivo multimode Raman imaging reveals concerted molecular composition and distribution changes during yeast cell cycle. Chem Commun. 2011;47(33):9423-5.

38. Heraud P, Marzec KM, Zhang QH, Yuen WS, Carroll J, Wood BR. Labelfree in vivo Raman microspectroscopic imaging of the macromolecular architecture of oocytes. Sci Rep. 2017;7:8945.

39. Perez-Guaita D, Kochan K, Martin M, Andrew DW, Heraud P, Richards JS, Wood SR. Multimodal vibrational imaging of cells. Vib Spectrosc. 2017;91:46-58.

40. Deniset-Besseau A, Prater CB, Virolle MJ, Dazzi A. Monitoring triacylglycerols accumulation by atomic force microscopy based infrared spectroscopy in Streptomyces species for biodiesel applications. J Phys Chem Lett. 2014:5(4):654-8.

41. Jamme F, Vindigni J-D, Méchin V, Cherifi T, Chardot T, Froissard M. Single cell synchrotron FT-IR microspectroscopy reveals a link between neutral lipid and storage carbohydrate fluxes in S. cerevisiae. PLOS ONE. 2013:8(9):e74421. 